\title{
THE ACTION ON COHOMOLOGY BY COMPOSITIONS OF RATIONAL MAPS
}

\author{
ROLAND K. W. ROEDER
}

\begin{abstract}
We use intuitive results from algebraic topology and intersection theory to clarify the pullback action on cohomology by compositions of rational maps. We use these techniques to prove a simple sufficient criterion for functoriality of a composition of two rational maps on all degrees of cohomology and we then reprove the criteria of Diller-Favre, Bedford-Kim, and Dinh-Sibony. We conclude with a cautionary example.
\end{abstract}

\section{INTRODUCTION}

Suppose that $X$ and $Y$ are complex projective algebraic manifolds, both of dimension $k$, and $f: X \rightarrow Y$ is a rational map. If $I_{f}$ denotes the indeterminacy set of $f$, the graph of $f$ is the irreducible variety

$$
\Gamma_{f}:=\overline{\left\{(x, y) \in X \times Y: x \notin I_{f} \text { and } y=f(x)\right\}} .
$$

One defines the action $f^{*}: H^{*}(Y) \rightarrow H^{*}(X)$ on the singular cohomology of $X$ by considering $f$ as the correspondence $\Gamma_{f} \subset X \times Y$. If $\pi_{1}: X \times Y \rightarrow X$ and $\pi_{2}: X \times Y \rightarrow Y$ are the canonical projections, then, for any $\alpha \in H^{i}(Y)$,

$$
f^{*} \alpha:=\pi_{1 *}\left(\left[\Gamma_{f}\right] \smile \pi_{2}^{*} \alpha\right) .
$$

Here, $\left[\Gamma_{f}\right]$ is the fundamental cohomology class of $\Gamma_{f}, \pi_{2}^{*}$ is the classical pullback on cohomology as defined for regular maps, and $\pi_{1 *}$ is the pushforward on cohomology, defined by $\pi_{1 *}=\mathrm{PD}_{X}^{-1} \circ \pi_{1 \#} \circ \mathrm{PD}_{X \times Y}$, where $\pi_{1 \#}$ denotes the push forward on homology and $\mathrm{PD}_{M}: H^{*}(M) \rightarrow H_{\operatorname{dim}_{\mathbb{R}} M-*}(M)$ denotes the Poincaré duality isomorphism on a manifold $M$. If $f$ is regular (i.e. $I_{f}=\emptyset$ ) then (2) coincides with the classical definition of pullback.

We will take the coefficients for our cohomology in $\mathbb{C}$, letting $H^{i}(X) \equiv H^{i}(X ; \mathbb{C})$. Since our manifolds are Kähler, there is a natural isomorphism

$$
\bigoplus_{p+q=i} H^{p, q}(X) \rightarrow H^{i}(X)
$$

where the former are the Dolbeault cohomology groups. This isomorphism induces a splitting of the singular cohomology of $X$ into bi-degrees, which one can check is invariant under the pullback (2).

The most primitive dynamical invariants of any rational selfmap $h: X \rightarrow X$ are the dynamical degrees

$$
\lambda_{p}(h):=\lim _{n \rightarrow \infty} \|\left(h^{n}\right)^{*}:\left.H^{p, p}(X) \rightarrow H^{p, p}(X)\right|^{1 / n},
$$

Date: October 17, 2018.

2010 Mathematics Subject Classification. Primary 37F99; Secondary 32H50.

Key words and phrases. pullback on cohomology, dynamical degrees. 
which are defined for $1 \leq p \leq k=\operatorname{dim}(X)$. They were introduced by Friedland [20] and by Russakovskii and Shiffman [33] and shown to be invariant under birational conjugacy by Dinh and Sibony [15]. Note that dynamical degrees were originally defined with the limit in (3) replaced by a limsup. However, it was shown in [15] that the limit always exists.

The dynamical degrees of $h$ are tied to the expected ergodic properties of $h$; see, for example, [24]. (These expected properties have been proved when $\lambda_{k}(h)$ is maximal $[25,14]$ or when $\operatorname{dim}(X)=2, \lambda_{1}(h)>\lambda_{2}(h)$, and certain minor technical hypotheses are satisfied [12].) Dynamical degrees are typically hard to compute because (2) does not behave well under composition of maps. There are simple examples for which $\left(h^{n}\right)^{*} \neq\left(h^{*}\right)^{n}$. One says that $h$ is $p$-stable if $\left(h^{n}\right)^{*}=\left(h^{*}\right)^{n}$ on $H^{p, p}(X)$ for every $n \in \mathbb{Z}^{+}$. A nice summary of techniques on how to compute dynamical degrees appears in [6]. Let us note that there are very few explicit examples $[2,18,31,32]$ in which the $p$-th dynamical degrees have been computed for $1<p<k$.

In order to study the problem of $p$-stability, one typically looks for criterion on $f$ : $X \rightarrow Y$ and $g: Y \rightarrow Z$ under which $(g \circ f)^{*}=f^{*} \circ g^{*}$ (either on all cohomology or for certain degrees). Such criteria have been given by Fornaess-Sibony [19], Diller-Favre [13], Bedford-Kim [7, 8], and Dinh-Sibony [16]. The proofs of these criteria typically represent a cohomology class $\alpha \in H^{*}(Z)$ with a smooth form, pull it back under $g^{*}$ as a closed current, and then pull back the resulting current under $f^{*}$. This approach is especially challenging when $p \geq 2$ since the pullback of such higher-codimension currents is very delicate.

The purpose of this note is to prove these criteria using intuitive techniques from cohomology and intersection theory. This approach is inspired by the techniques used by Amerik in [2]. Our primary motivation is to provide those who are learning these results with an alternative approach, in the hope that seeing two different proofs makes the results clearer.

Another merit of this approach is that it may be possible to adapt it to problems about rational maps between projective manifolds defined over fields $K \neq \mathbb{C}$. The dynamics of such mappings has gained considerable interest recently (see, for example, [3, 27, 28, 36, 34] and the references therein) and the analytic techniques involving smooth forms and positive closed currents from $[13,7,8,16]$ do not apply in that context. However, Intersection Theory (our main underlying tool) still applies to projective manifolds defined over other fields $K$.

Several of the references listed above consider the broader context of meromorphic maps of compact Kähler manifolds. In order for the techniques used in this note to be as elementary as possible, we will restrict our attention to rational maps of projective algebraic manifolds. This allows us to use classical techniques from intersection theory, such as Fulton's Excess Intersection Formula, which will be helpful when establishing Lemma 2.5, below.

Let us make the convention that all rational maps are dominant, meaning that the image is not contained within a proper subvariety of the codomain. To be concise, we will use the term algebraic manifold to mean complex projective algebraic manifold. Moreover, since we are primarily motivated by dynamics, all rational mappings will be between algebraic manifolds of the same dimension. For any $S \subset X$, we define $f(S):=\pi_{2}\left(\pi_{1}^{-1}(S) \cap \Gamma_{f}\right)$ and for any $S \subset Y$ we define $f^{-1}(S):=\pi_{1}\left(\pi_{2}^{-1}(S) \cap \Gamma_{f}\right)$

For simplicity of exposition, we will ignore the decomposition of cohomology into bidegree wherever possible. 
In order to study the composition $g \circ f$ we will need the following diagram:

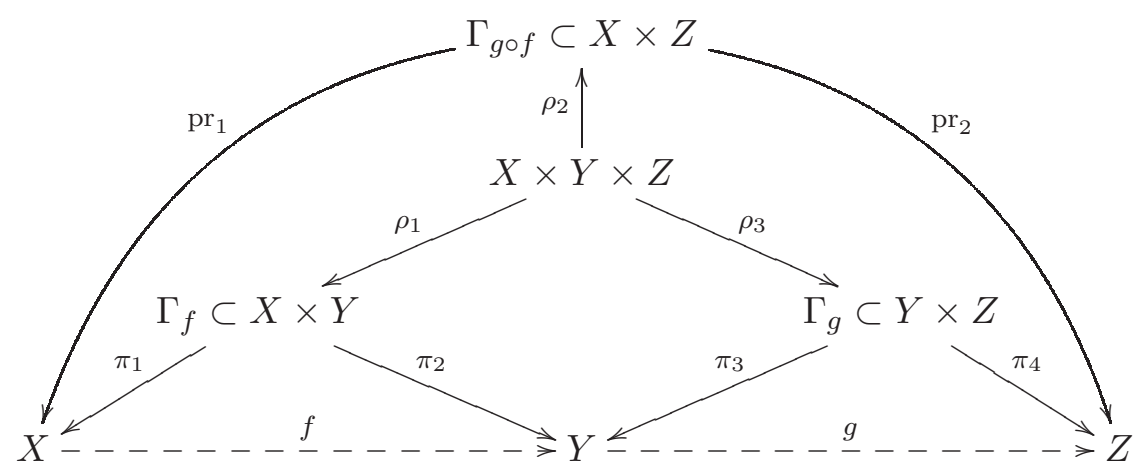

Central to the entire discussion is the following.

Proposition 1.1. We have

$$
f^{*} g^{*} \alpha=\operatorname{pr}_{1 *}\left(\rho_{2 *}\left(\rho_{1}^{*}\left[\Gamma_{f}\right] \smile \rho_{3}^{*}\left[\Gamma_{g}\right]\right) \smile \operatorname{pr}_{2}^{*} \alpha\right) .
$$

In particular, $(g \circ f)^{*}=f^{*} \circ g^{*}$ on all cohomology groups if and only if

$$
\left[\Gamma_{g \circ f}\right]=\rho_{2 *}\left(\rho_{1}^{*}\left[\Gamma_{f}\right] \smile \rho_{3}^{*}\left[\Gamma_{g}\right]\right) \in H^{2 k}(X \times Z) .
$$

This proposition is probably well-known within algebraic geometry, for example a variant of (5) is proved for the pull back on the Chow Ring in [22, Sec. 16.1] and [38, Prop. 9.7], but it seems to be less well-known in rational dynamics.

Our first application of Proposition 1.1 is to prove:

Proposition 1.2. Let $f: X \rightarrow Y$ and $g: Y \rightarrow Z$ be rational maps. Suppose that there exits an algebraic manifold $\widetilde{X}$ and holomorphic maps pr and $\tilde{f}$ making the following diagram commute (wherever $f \circ \mathrm{pr}$ is defined)

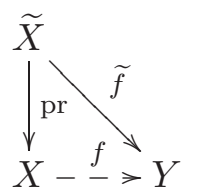

with the property that $\widetilde{f}^{-1}(x)$ is a finite set for every $y \in Y$. Then, $(g \circ f)^{*}=f^{*} \circ g^{*}$ on all cohomology groups.

Remark 1.3. In many cases, $\widetilde{X}$ will be a blow-up of $X$. However this is not a hypothesis of Proposition 1.2, which can also be useful in other situations. Notice also that the condition that $\widetilde{f}^{-1}(x)$ is a finite set implies that $\operatorname{dim}(\widetilde{X})=k$.

After proving Proposition 1.2, we will use Proposition 1.1 to prove the criteria of DillerFavre, Bedford-Kim, and Dinh-Sibony stated below.

Historically, the first criterion for functoriality of pullbacks under compositions was given by Fornaess and Sibony [19] who proved that if $f: \mathbb{C P}^{k} \rightarrow \mathbb{C P}^{k}$ and $g: \mathbb{C P}^{k} \rightarrow \mathbb{C P}^{k}$ are rational maps then $(g \circ f)^{*}=f^{*} \circ g^{*}$ on the second cohomology if and only if there is no hypersurface $H \subset \mathbb{P}^{k}$ with $f\left(H \backslash I_{f}\right) \subset I_{g}$. The proof consists of recognizing that the homogeneous expression obtained when composing $f$ and $g$ has a common factor of positive degree if and only if there is a hypersurface $H \subset \mathbb{P}^{k}$ with $f\left(H \backslash I_{f}\right) \subset I_{g}$. Since this common factor must be removed in order to define $g \circ f$, the resulting composition has lower degree. 
A further study of this phenomenon and a characterization of the sequences of degrees that may appear for the iterates of such a map $f$ is given in [9].

Since $I_{g}$ is of codimension at least two, in order that $f\left(H \backslash I_{f}\right) \subset I_{g}, f$ must collapse $H$ to a variety of lower dimension. The principle that non-functoriality is caused by collapse of a subvariety under $f$ to something of lower dimension that is contained within $I_{g}$ appears as a common theme in the following three criteria:

Proposition 1.4. (Diller-Favre [13, Prop. 1.13]) Let $X, Y$, and $Z$ be algebraic manifolds of dimension 2. Let $f: X \rightarrow Y$ and $g: Y \rightarrow Z$ be rational maps. Then $(g \circ f)^{*}=f^{*} \circ g^{*}$ if and only if there is no curve $C \subset X$ with $f\left(C \backslash I_{f}\right) \subset I_{g}$.

Proposition 1.5. (Bedford-Kim [8, Thm. 1.1]) Let $X, Y$, and $Z$ be algebraic manifolds of dimension $k$. Let $f: X \rightarrow Y$ and $g: Y \rightarrow Z$ be rational maps. If there is no hypersurface $H$ with $f\left(H \backslash I_{f}\right) \subset I_{g}$, then $f^{*} \circ g^{*}=(g \circ f)^{*}$ on $H^{2}(Z)$.

We will prove a slightly stronger variant of the criterion of Dinh and Sibony. Let $\widetilde{\Sigma}_{f}^{\prime} \subset \Gamma_{f}$ is the set of points such that

(i) $\pi_{2}$ restricted to $\Gamma_{f}$ is not locally finite at $x$, and

(ii) $\pi_{2}((x, y)) \in I_{g}$ for every $(x, y) \in \widetilde{\Sigma}_{f}^{\prime}$.

Let $\Sigma_{f}^{\prime}:=\pi_{1}\left(\widetilde{\Sigma}_{f}^{\prime}\right)$.

Proposition 1.6. (Variant of Dinh-Sibony [16, Prop. 5.3.5]) Let $X, Y$, and $Z$ be algebraic manifolds of dimension $k$. Let $f: X \rightarrow Y$ and $g: Y \rightarrow Z$ be rational maps. If $\operatorname{dim} \Sigma_{f}^{\prime}<$ $k-p$, then $(g \circ f)^{*}=f^{*} \circ g^{*}$ on $H^{i}(Z)$ for $1 \leq i \leq 2 p$.

Remark 1.7. The distinction between this criterion and the one from [16, Prop. 5.3.5] is that we impose the extra condition (ii) on $\Sigma_{f}$, allowing for higher dimensional varieties to be collapsed by $f$, so long as they don't map into $I_{g}$.

Remark 1.8. Proposition 1.2, the sufficiency condition in Proposition 1.4, and Proposition 1.5 can all be obtained as corollaries to Proposition 1.6. However, we'll present them separately since they're of independent interest and their direct proofs are simpler.

In $\S 2$ we provide a brief background with needed tools from cohomology and intersection theory. In $\S 3$ we discuss some further properties of the graph $\Gamma_{f}$ and we show that definition (2) of $f^{*}$ is equivalent with some of the other standard versions appearing in the literature. We prove Propositions 1.1 in $\S 4$. In $\S 5$ we prove Propositions 1.2-1.6. This paper is concluded with $\S 6$ in which we provide a cautionary example, presenting a rational map $f: X \rightarrow X$ of a three dimensional manifold $X$ that is not 2-stable but has the property that $\left(\left.f\right|_{X \backslash I_{f}}\right)^{-1}(x)$ is finite for every $x \in X$. This example illustrates that to study $p$ stability for $1<p<k$, one must consider collapsing behavior lying within the indeterminate set.

\section{BACKGROUND FROM COHOMOLOGY AND INTERSECTION THEORY}

Suppose $f: M \rightarrow N$ is a continuous map between compact manifolds of dimensions $m$ and $n$, respectively. Given $\alpha \in H^{i}(M)$, we define $f_{*}: H^{i}(M) \rightarrow H^{n-m+i}(N)$ by

$$
f_{*} \alpha:=\mathrm{PD}_{N}^{-1}\left(f_{\#}\left(\mathrm{PD}_{M} \alpha\right)\right)
$$

where $f_{\#}: H_{*}(M) \rightarrow H_{*}(N)$ is the push forward on homology.

We will make extensive use of the following formula. 
Lemma 2.1 (Push-Pull Formula). Suppose $M$ and $N$ are manifolds and $f: M \rightarrow N$ is continuous. Then, for any $\alpha \in H^{i}(N)$ and any $\beta \in H^{j}(M)$ we have

$$
f_{*}\left(f^{*}(\alpha) \smile \beta\right)=\alpha \smile f_{*}(\beta) \in H^{n-m+i+j}(N) .
$$

Note that when $f$ is holomorphic, this is sometimes also called the "projection formula".

Proof. This is a simple consequence of the following three facts

(i) Push-Pull formula on homology: If $f: M \rightarrow N$ is continuous, $\eta \in H^{*}(N)$, and $\gamma \in H_{*}(M)$, then

$$
f_{\#}\left(f^{*}(\eta) \frown \gamma\right)=\eta \frown f_{\#} \gamma
$$

(ii) $\operatorname{PD}_{M}(\alpha)$ is defined by $\alpha \frown\{M\}$, where $\{M\}$ is the fundamental homology class of $M$, and

(iii) for any $\eta, \phi \in H^{*}(M)$ and $\gamma \in H_{*}(M)$, then $(\eta \smile \phi) \frown \gamma=\eta \frown(\phi \frown \gamma)$.

See [10, Ch. VI, Thm. 5.1 and Cor. 9.3].

We will need a little bit of information about the Künneth formuli on cohomology and homology. Recall that our (co)homology is taken with coefficients in the field $\mathbb{C}$. Let

$$
\kappa_{i}: \bigoplus_{a+b=i} H^{a}(M) \otimes H^{b}(N) \rightarrow H^{i}(M \times N)
$$

and

$$
K_{i}: \bigoplus_{a+b=i} H_{a}(M) \otimes H_{b}(N) \rightarrow H_{i}(M \times N)
$$

be the Künneth isomorphisms. Recall that $\kappa_{i}(\gamma \otimes \eta)=\pi_{1}^{*} \gamma \smile \pi_{2}^{*} \eta$. Suppose $M$ and $N$ are manifolds.

Lemma 2.2. The following diagram commutes:

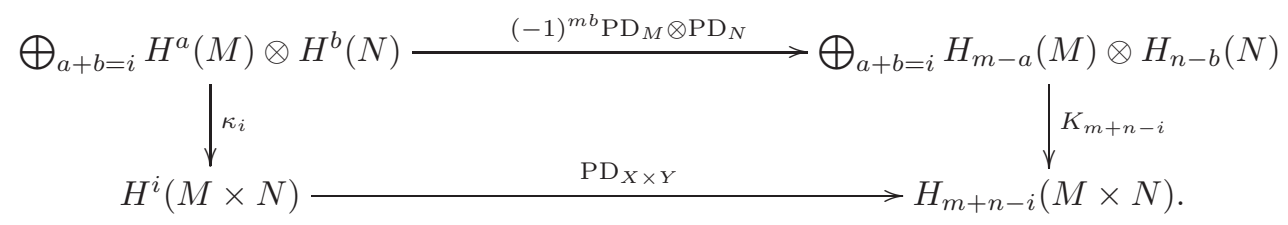

Proof. According to [10, Ch. VI, Thm. 5.4], if $\alpha \in H^{*}(X), \beta \in H^{*}(Y), c \in H_{*}(X)$, and $d \in H_{*}(Y)$, then

$$
\kappa(\alpha \otimes \beta) \frown K(c \otimes d)=(-1)^{\operatorname{deg}(\beta) \operatorname{deg}(c)} K((\alpha \frown c) \otimes(\beta \frown d)) .
$$

The result follows, since $\mathrm{PD}_{M \times N}$ is obtained by taking the cap product with $\{M \times N\}$ $=K(\{M\} \otimes\{N\})$.

Lemma 2.3. Let $M$ and $N$ be connected manifolds of dimensions $m$ and $n$, respectively and let $\pi: M \times N \rightarrow M$ be projection onto the first coordinate. Suppose $\nu \in H^{i}(M \times N)$ satisfies

$$
\kappa^{-1}(\nu)=\sum_{a=1}^{i} \sum_{l=1}^{l_{j}} \gamma_{i-a, l} \otimes \eta_{a, l}
$$


with $\gamma_{i-a, l} \in H^{i-a}(M), \eta_{a, l} \in H^{a}(N)$, and with the normalization that each $\eta_{n, l} \in H^{n}(N)$ is the fundamental class $[x]$ of a point $x \in N$. Then

$$
\pi_{*} \nu=(-1)^{m n} \sum_{l=1}^{l_{n}} \gamma_{i-n, l} .
$$

Proof. This follows from Lemma 2.2 and the fact that the push forward $\mathrm{pr}_{\#}$ on homology satisfies that

$$
\operatorname{pr}_{\#}\left(K\left(\sum_{a=1}^{i} \sum_{l=1}^{l_{a}} g_{i-a, l} \otimes e_{a, l}\right)\right)=\sum_{l=1}^{l_{0}} g_{i, l},
$$

if each $g_{i-a, l} \in H_{i-a}(M)$, each $e_{a, l} \in H_{a}(N)$, and each $e_{0, i}=\{x\}$ is the fundamental homology class of a point. This follows easily from the fact that the Künneth Isomorphism is natural with respect to induced maps.

Remark 2.4. In our applications, $M$ and $N$ will be complex manifolds. Since they have even real-dimension, the signs will disappear from Lemmas 2.2 and 2.3.

Let $X$ be an algebraic manifold of (complex) dimension $k$ and let $V \subset X$ be a subvariety of dimension $k-i$. It is well known that $V$ generates a cohomology class $[V] \in H^{2 i}(X)$; see, for example, [23,37]. If $V^{\prime} \subset X$ is another subvariety of dimension $k-j$, we will need information relating $[V] \smile\left[V^{\prime}\right]$ to $V \cap V^{\prime}$. This is the subject of Intersection Theory $[21,22,38]$. One says that $V$ and $V^{\prime}$ are transverse at generic points of $V \cap V^{\prime}$ if there is a dense set of $V \cap V^{\prime}$ on which $V$ and $V^{\prime}$ are both smooth and intersect transversally. The information we need is encapsulated in:

Lemma 2.5. Let $V$ and $V^{\prime}$ be subvarieties of $X$ of dimensions $k-i$ and $k-j$. Then, $[V] \smile\left[V^{\prime}\right]$ is represented as a linear combination of fundamental cohomology classes of $k-i-j$-dimensional subvarieties of $V \cap V^{\prime}$. More specifically:

(i) If $V$ and $V^{\prime}$ are transverse at generic points of $V \cap V^{\prime}$, then $[V] \smile\left[V^{\prime}\right]=\left[V \cap V^{\prime}\right]$.

(ii) More generally, if each of the components $W_{1}, \ldots, W_{m_{0}}$ of $V \cap V^{\prime}$ has the correct dimension of $k-i-j$, then

$$
[V] \smile\left[V^{\prime}\right]=\sum_{m=1}^{m_{0}} a_{m}\left[W_{m}\right],
$$

where each $a_{m} \in \mathbb{Z}^{+}$is an intersection number satisfying that $a_{m}=1$ if and only if $V$ and $V^{\prime}$ are transverse at generic points of $W_{m}$.

(iii) Most generally, if some of the components $W_{1}, \ldots, W_{m_{0}}$ of $V \cap V^{\prime}$ are of dimension $>k-i-j$, then,

$$
[V] \smile\left[V^{\prime}\right]=\sum_{m=1}^{m_{0}} \sum_{n=1}^{n_{m}} a_{m, n}\left[W_{m, n}\right],
$$

where each $W_{m, n} \subset W_{m}$ is a subvariety of $W_{m}$ of dimension $k-i-j$ and each $a_{m, n} \in \mathbb{Z}$. For each $W_{m}$ of the correct dimension $k-i-j$ the inner sum reduces to be $a_{m}\left[W_{m}\right]$, where $a_{m}$ is given as in (ii).

Note that in case (iii), the coefficients $a_{m, n}$ can be negative, for example the selfintersection of the exceptional divisor resulting from a blow-up of $\mathbb{C P}^{2}$ is represented by a single point on the exceptional divisor with coefficient -1 . 
Rather than presenting a proof of Lemma 2.5, we will mention how to obtain it from the corresponding properties in the Chow Ring $C H^{*}(X)$, which are proved in $[21,22,38]$. For each $0 \leq i \leq k$, the chow group $C H^{i}(X)$ is the collection of finite formal sums of $k-i$ dimensional irreducible subvarieties taken with integer coefficients, up to an equivalence relation known as rational equivalence. We won't need the detailed definition of rational equivalence, however let us denote the rational equivalence class of an irreducible subvariety $V$ by $(V)$.

One obtains the Chow Ring $C H^{*}(X)=\bigoplus_{i=0}^{k} C H^{i}(X)$ by defining an intersection product

$$
\text { - : } C H^{i}(X) \times C H^{j}(X) \rightarrow C H^{i+j}(X) \text {. }
$$

If $V$ and $V^{\prime}$ intersect properly, with dimension $k-i-j$, then each component of the intersection is assigned an intersection multiplicity in a relatively simple way, see [22, Sec. 8.2]. (Note that using the uniqueness described in [22, Eg. 11.4.1], one can show that this intersection multiplicity is consistent with the more intuitive approach of [11, Sec. 12.3].) This intersection multiplicity is a positive integer that equals 1 if and only if $V$ and $V^{\prime}$ are generically transverse along the component.

If the intersection has a component whose dimension is larger than $k-i-j$, there are two approaches:

(i) moving one of the subvarieties $V$ to a rationally equivalent one $\widetilde{V}$ in such a way that $\widetilde{V} \cap V^{\prime}$ has the correct dimension, via Chow's moving lemma (see [38, Lem. 9.22] or [22, Sec. 11.4]), or

(ii) or Fulton's excess intersection formula, which represents the intersection product as a linear combination of subvarieties lying within $V \cap V^{\prime}$ (see [38, Sec. 9.2] or [22, Sec. 6.3]).

In order to guarantee the property that the cup product is represented by a sum of fundamental classes of subvarieties of $V \cap V^{\prime}$, we appeal to the latter.

Lemma 2.5 then follows from the fact that there is a ring homomorphism cl :CH* $(X) \rightarrow$ $H^{2 *}(X)$ with the property that for any irreducible $V \subset X, \operatorname{cl}((V))=[V]$. See, for example, [22, Ch. 19] or [38, Lem. 9.18 and Prop. 9.20].

Remark 2.6. In many of our applications, we will only need properties (i) and (ii) which are relatively simple. We will only use property (iii) to show that the cup product is given by subvarieties of the geometric intersection $V \cap V^{\prime}$. We won't use any details of how the coefficients $a_{m, n}$ in Part (iii) of Lemma 2.5 are actually computed.

Lemma 2.7. Suppose that $f: X \rightarrow Y$ is a proper holomorphic map between algebraic manifolds. For any irreducible subvariety $V \subset X$ we have

(i) if $\operatorname{dim}(f(V))=\operatorname{dim}(V)$, then $f_{*}([V])=\operatorname{deg}_{\text {top }}\left(\left.f\right|_{V}\right)[f(V)]$, where $\operatorname{deg}_{\text {top }}\left(\left.f\right|_{V}\right)$ is the number of preimages under $\left.f\right|_{V}$ of a generic point from $f(V)$.

(ii) Otherwise, $f_{*}([V])=0$.

Proof. This is essentially [22, Lem. 19.1.2] combined the remark in Section 1.4 of [22] that $\operatorname{deg}(V / f(V))$ is equal to the topological degree $\operatorname{deg}_{\text {top }}\left(\left.f\right|_{V}\right)$ of $\left.f\right|_{V}: V \rightarrow f(V)$.

Lemma 2.8. Let $X$ and $Z$ be $k$-dimensional algebraic manifolds and let $W$ be a $k$-dimensional subvariety of $X \times Z$. We have

$$
\operatorname{pr}_{1 *}\left([W] \smile \operatorname{pr}_{2}^{*} \alpha\right)=0
$$


if either

(i) $\operatorname{dim}\left(\operatorname{pr}_{1}(W)\right) \leq k-p$ and $\alpha \in H^{i}(Z)$ for some $i<2 p$, or

(ii) $\operatorname{dim}\left(\operatorname{pr}_{2}(W)\right) \leq p$ and $\alpha \in H^{i}(Z)$ for some $i>2 p$.

Proof. Suppose $\operatorname{dim}\left(\operatorname{pr}_{1}(W)\right) \leq k-p$. The fundamental homology class $\{W\}$ is in the image of $\iota_{\#}$, where $\iota: \operatorname{pr}_{1}(W) \times Z \hookrightarrow X \times Z$ is the inclusion. Therefore,

$$
K^{-1}(\{W\})=\sum_{a=1}^{2 k-2 p} \sum_{l=1}^{l_{a}} g_{a, l} \otimes e_{2 k-a, l},
$$

with each $g_{a, l} \in H_{a}(X)$ and each $e_{2 k-a, l} \in H_{2 k-a}(Z)$. Applying Lemma 2.2, we have

$$
\kappa^{-1}([W])=\sum_{a=1}^{2 k-2 p} \sum_{l=1}^{l_{a}} \operatorname{PD}_{X}^{-1}\left(g_{a, l}\right) \otimes \operatorname{PD}_{Y}^{-1}\left(e_{2 k-a, l}\right)=\sum_{a=1}^{2 k-2 p} \sum_{l=1}^{l_{a}} \gamma_{2 k-a, l} \otimes \eta_{a, l} .
$$

where each $\gamma_{2 k-a, l} \in H^{2 k-a}(X)$ and $\eta_{a, l} \in H^{a}(Z)$. Thus for any $\alpha \in H^{i}(Z)$ we have,

$$
\begin{aligned}
{[W] \smile \operatorname{pr}_{2}^{*} \alpha } & =\sum_{a=1}^{2 k-2 p} \sum_{l=1}^{l_{a}} \operatorname{pr}_{1}^{*}\left(\gamma_{2 k-a, l}\right) \smile \operatorname{pr}_{2}^{*}\left(\eta_{a, l} \smile \alpha\right) \\
& =\kappa^{-1}\left(\sum_{a=1}^{2 k-2 p} \sum_{l=1}^{l_{a}} \gamma_{2 k-a, l} \otimes\left(\eta_{a, l} \smile \alpha\right)\right) .
\end{aligned}
$$

Since each term in the second factor has degree $2 k-2 p+i<2 k$, Lemma 2.3 gives that

$$
\operatorname{pr}_{2 *}\left([W] \smile \operatorname{pr}_{2}^{*} \alpha\right)=0 .
$$

The proof of (ii) is essentially the same.

\section{Alternative definitions for $f^{*}$ and Remarks about $\Gamma_{f}$}

In this section, we'll show that two common alternative definitions for $f^{*} \alpha$ are consistent with (2). In Example 3.4, we'll see that the graph $\Gamma_{f}$ may be singular at points whose first coordinate is in $I_{f}$. For this reason, these alternative definitions for $f^{*} \alpha$ are more commonly used in actual computations.

Lemma 3.1. Suppose $\widetilde{X}$ is a k-dimensional algebraic manifold and that $\operatorname{pr}: \widetilde{X} \rightarrow X$ and $\tilde{f}$ : $\widetilde{X} \rightarrow Y$ are holomorphic maps making the Diagram (6) commute. Then, $f^{*} \alpha=\operatorname{pr}_{*}\left(\tilde{f}^{*} \alpha\right)$.

Usually, pr: $\widetilde{X} \rightarrow X$ will be a blow-up, but Lemma 3.1 holds in greater generality.

Proof. This follows from the fact that $(\operatorname{pr} \times \mathrm{id})_{*}\left[\Gamma_{\widetilde{f}}\right]=\left[\Gamma_{f}\right]$ and the Push-Pull formula.

Lemma 3.2. Suppose that $\widetilde{\Gamma_{f}}$ is a resolution of the singularities in $\Gamma_{f}$ and $\widetilde{\pi_{1}}$ and $\widetilde{\pi_{2}}$ are the lifts of $\left.\pi_{1}\right|_{\Gamma_{f}}$ and $\left.\pi_{2}\right|_{\Gamma_{f}}$ to $\widetilde{\Gamma_{f}}$. Then, $f^{*} \alpha=\widetilde{\pi}_{1 *}\left(\widetilde{\pi}_{2}^{*} \alpha\right)$.

Proof. This is a restatement of Lemma 3.1.

The following lemma will be helpful later.

Lemma 3.3. Let $X$ and $Y$ be algebraic manifolds of dimension $k$ and let $f: X \rightarrow Y$ be $a$ rational map. If $V \subset X$ be a proper subvariety of $X$, then

$$
\Gamma_{f}=\overline{\left\{(x, y) \in X \times Y: x \notin V \cup I_{f} \text { and } y=f(x)\right\}} .
$$


Proof. Since $\Gamma_{f}$ is defined by (1), it suffices to show that $\Gamma_{f} \backslash \pi_{1}^{-1}(V)$ is dense in $\Gamma_{f}$. This follows since $\pi_{1}: \Gamma_{f} \rightarrow X$ is dominant, giving that $\pi_{1}^{-1}(V)$ is a proper subvariety of $\Gamma_{f}$.

Example 3.4. Both Eric Bedford and the one of the anonymous referees have pointed out to us several complications arising when working directly with the graph $\Gamma_{f}$. The following is an expanded version of Example 1 from $[5, \S 5]$.

The quadratic Hénon map $h_{a, c}: \mathbb{C}^{2} \rightarrow \mathbb{C}^{2}$, given by

$$
h_{a, c}\left(x_{1}, x_{2}\right)=\left(x_{1}^{2}+c-a x_{2}, x_{1}\right),
$$

extends as a birational map of $\mathbb{P}^{2}$, which is expressed in homogeneous coordinates as

$$
h_{a, c}\left(\left[X_{1}: X_{2}: X_{3}\right]\right)=\left[X_{1}^{2}+c X_{3}^{2}-a X_{2} X_{3}: X_{1} X_{3}: X_{3}^{2}\right] .
$$

The extension has indeterminacy $I_{h}=\{[0: 1: 0]\}$. One can check that $I_{h}$ blows-up under $h$ to $L_{\infty}:=\left\{X_{3}=0\right\}$ and that $h\left(L_{\infty} \backslash I_{h}\right)=[1: 0: 0]$. In particular, points of $\Gamma_{h}$ satisfy that $X_{3}=0 \Leftrightarrow Y_{3}=0$.

In $\mathbb{C}^{2} \times \mathbb{C}^{2}$, the graph of $h$ is given by

$$
y_{1}=x_{1}^{2}+c-a x_{2} \quad \text { and } \quad y_{2}=x_{1} .
$$

It is natural to expect that the graph $\Gamma_{h}$ of $h: \mathbb{P}^{2} \rightarrow \mathbb{P}^{2}$ is obtained by substituting $x_{1}=\frac{X_{1}}{X_{3}}, x_{2}=\frac{X_{2}}{X_{3}}, y_{1}=\frac{Y_{1}}{Y_{3}}$, and $y_{2}=\frac{Y_{2}}{Y_{3}}$ and then clearing denominators. One obtains

$$
\begin{aligned}
& Y_{1} X_{3}^{2}=X_{1}^{2} Y_{3}+c X_{3}^{2} Y_{3}-a X_{2} X_{3} Y_{3} \quad \text { and } \\
& Y_{2} X_{3}=X_{1} Y_{3},
\end{aligned}
$$

which describe some subset of $\mathbb{P}^{2} \times \mathbb{P}^{2}$. However, if one sets $X_{3}=Y_{3}=0$, both equations become $0=0$, so that $\left[X_{1}: X_{2}: 0\right] \times\left[Y_{1}: Y_{2}: 0\right]$ satisfies (12) for any $X_{1}, X_{2}, Y_{1}$, and $Y_{2}$. Thus, (12) does not capture the fact that $h\left(L_{\infty} \backslash I_{h}\right)=[1: 0: 0]$.

One can try adding further equations that are consistent with (11) on $\mathbb{C}^{2} \times \mathbb{C}^{2}$. When $X_{3}$ and $Y_{3}$ are not 0 , it follows from the second equation in (12) that $\frac{X_{1}}{X_{3}}=\frac{Y_{2}}{Y_{3}}$, which implies $\frac{1}{X_{3}}=\frac{Y_{2}}{Y_{3} X_{1}}$. Substituting $x_{1}=\frac{X_{1} Y_{2}}{Y_{3} X_{1}}=\frac{Y_{2}}{Y_{3}}, x_{2}=\frac{X_{2} Y_{2}}{Y_{3} X_{1}}$ and $y_{1}=\frac{Y_{1}}{Y_{3}}$ into first equation from (11), clearing denominators, and dividing by a common factor of $X_{1}$ adds a third equation to the system:

$$
\begin{aligned}
Y_{1} X_{3}^{2} & =X_{1}^{2} Y_{3}+c X_{3}^{2} Y_{3}-a X_{2} X_{3} Y_{3}, \\
Y_{2} X_{3} & =X_{1} Y_{3}, \quad \text { and } \\
Y_{1} Y_{3} X_{1} & =X_{1} Y_{2}^{2}+c Y_{3}^{2} X_{1}-a X_{2} Y_{2} Y_{3} .
\end{aligned}
$$

When one substitutes $X_{3}=Y_{3}=0$ into (13), the third equation becomes $0=X_{1} Y_{2}^{2}$, which expresses that $h\left(L_{\infty} \backslash I_{h}\right)=[1: 0: 0]$. However, $[0: 1: 0] \times\left[Y_{1}: 0: Y_{3}\right]$ satisfies (13) for any $Y_{1}$ and $Y_{3}$. Thus (13) does not imply that $X_{3}=0 \Leftrightarrow Y_{3}=0$, which is required for points of $\Gamma_{h}$.

If one computes $h^{-1}: \mathbb{C}^{2} \rightarrow \mathbb{C}^{2}$, one of the equations is $x_{2}=\frac{1}{a}\left(y_{2}^{2}+c-y_{1}\right)$. Converting this equation to homogeneous coordinates and adding it to our system, we obtain

$$
\begin{aligned}
Y_{1} X_{3}^{2} & =X_{1}^{2} Y_{3}+c X_{3}^{2} Y_{3}-a X_{2} X_{3} Y_{3}, \\
Y_{2} X_{3} & =X_{1} Y_{3}, \\
Y_{1} Y_{3} X_{1} & =X_{1} Y_{2}^{2}+c Y_{3}^{2} X_{1}-a X_{2} Y_{2} Y_{3}, \quad \text { and } \\
a X_{2} Y_{3}^{2} & =X_{3} Y_{2}^{2}+c X_{3} Y_{3}^{2}-Y_{1} Y_{3} X_{3} .
\end{aligned}
$$


These four equations imply that $X_{3}=0 \Leftrightarrow Y_{3}=0$. When $X_{3}=Y_{3}=0$, the third equation becomes $0=X_{1} Y_{2}^{2}$, which describes $\Gamma_{h} \cap\left(L_{\infty} \times L_{\infty}\right)$ and when $X_{3} \neq 0$ and $Y_{3} \neq 0$ the first two equations describe $\Gamma_{h} \cap\left(\mathbb{C}^{2} \times \mathbb{C}^{2}\right)$. Therefore, (14) describes $\Gamma_{h} \subset \mathbb{P}^{2} \times \mathbb{P}^{2}$.

One might wonder whether $\Gamma_{h}$ can be described with fewer equations. This is related to the notion on complete intersection; see, for example, [26, Exercise I.2.17]. If we let $J$ be the ideal in $\mathbb{C}\left[X_{1}, X_{2}, X_{3}, Y_{1}, Y_{2}, Y_{3}\right]$ defined by (14), one can compute $I\left(\Gamma_{h}\right)=\sqrt{J}$ using the computer algebra package Macaulay2 [1]. One finds that $I\left(\Gamma_{h}\right)$ is generated by two equations

$$
\begin{aligned}
X_{3} Y_{2}-X_{1} Y_{3} & =0, \quad \text { and } \\
X_{3} Y_{1}-X_{1} Y_{2}+a X_{2} Y_{3}-c X_{3} Y_{3} & =0 .
\end{aligned}
$$

Thus, $\Gamma_{h}$ is a complete intersection. In particular, these two equations describe $\Gamma_{h}$ in all of $\mathbb{P}^{2} \times \mathbb{P}^{2}$.

If we express (15) in the local coordinates $z_{1}=X_{1} / X_{2}, z_{2}=X_{3} / X_{2}, w_{1}=Y_{2} / Y_{1}$, and $w_{2}=Y_{3} / Y_{1}$ centered at $[0: 1: 0] \times[1: 0: 0]$, we find

$$
\begin{array}{rlll}
z_{2} w_{1}-z_{1} w_{2} & = & 0 & \text { and } \\
z_{2}-z_{1} w_{1}+a w_{2}-c z_{2} w_{2} & = & 0 .
\end{array}
$$

Since the lowest order terms of the first equation are quadratic, when one restricts $\Gamma_{h}$ to any plane through $\left(z_{1}, z_{2}, w_{1}, w_{2}\right)=(0,0,0,0)$, the result will have local multiplicity $\geq 2$. This implies that $\Gamma_{h}$ has local multiplicity $\geq 2$ at $[0: 1: 0] \times[1: 0: 0]$ and hence that $\Gamma_{h}$ is singular there.

\section{Proof of the Composition Formula}

The proof of (5) below is cribbed from Voisin's textbook [38, Prop. 9.17].

Proof. Proof of Proposition 1.1: For any $\alpha \in H^{*}(Z)$ we have

$$
\begin{aligned}
\operatorname{pr}_{1 *}\left(\rho_{2 *}\left(\rho_{1}^{*}\left[\Gamma_{f}\right] \smile \rho_{3}^{*}\left[\Gamma_{g}\right]\right) \smile \operatorname{pr}_{2}^{*} \alpha\right) & \stackrel{\text { PP }}{=} \operatorname{pr}_{1 *}\left(\rho_{2 *}\left(\rho_{1}^{*}\left[\Gamma_{f}\right] \smile \rho_{3}^{*}\left[\Gamma_{g}\right] \smile \rho_{2}^{*} \operatorname{pr}_{2}^{*} \alpha\right)\right) \\
=\operatorname{pr}_{1 *}\left(\rho_{2 *}\left(\rho_{1}^{*}\left[\Gamma_{f}\right] \smile \rho_{3}^{*}\left[\Gamma_{g}\right] \smile\left(\pi_{4} \circ \rho_{3}\right)^{*} \alpha\right)\right) & =\pi_{1 *}\left(\rho_{1 *}\left(\rho_{1}^{*}\left[\Gamma_{f}\right] \smile \rho_{3}^{*}\left[\Gamma_{g}\right] \smile\left(\pi_{4} \circ \rho_{3}\right)^{*} \alpha\right)\right) \\
\stackrel{\mathrm{PP}}{=} \pi_{1 *}\left(\left[\Gamma_{f}\right] \smile \rho_{1 *}\left(\rho_{3}^{*}\left[\Gamma_{g}\right] \smile\left(\pi_{4} \circ \rho_{3}\right)^{*} \alpha\right)\right) & =\pi_{1 *}\left(\left[\Gamma_{f}\right] \smile \rho_{1 *}\left(\rho_{3}^{*}\left(\left[\Gamma_{g}\right] \smile \pi_{4}^{*} \alpha\right)\right)\right) \\
\stackrel{\triangleq}{=} \pi_{1 *}\left(\left[\Gamma_{f}\right] \smile \pi_{2}^{*}\left(\pi_{3 *}\left(\left[\Gamma_{g}\right] \smile \pi_{4}^{*} \alpha\right)\right)\right) & =f^{*} g^{*} \alpha .
\end{aligned}
$$

Here, all unlabeled equalities follow from commutativity of Diagram (4) and the equality labeled PP follows from the Push-Pull formula. To check $\diamond$, one must show for any $\beta \in$ $H^{*}(Y \times Z)$ that

$$
\left(\rho_{1 *} \circ \rho_{3}^{*}\right) \beta=\left(\pi_{2}^{*} \circ \pi_{3 *}\right) \beta .
$$

This follows easily by expanding $\beta$ using the Künneth formula and applying Lemma 2.3.

We'll now check that if $\rho_{2 *}\left(\rho_{1}^{*}\left[\Gamma_{f}\right] \smile \rho_{3}^{*}\left[\Gamma_{g}\right]\right) \neq\left[\Gamma_{g \circ f}\right]$, then $f^{*} g^{*} \neq(g \circ f)^{*}$. Let

$$
\rho_{2 *}\left(\rho_{1}^{*}\left[\Gamma_{f}\right] \smile \rho_{3}^{*}\left[\Gamma_{g}\right]\right)=\left[\Gamma_{g \circ f}\right]+\mathcal{E} .
$$

By linearity of (5), it suffices to find some $\alpha \in H^{*}(Z)$ with $\operatorname{pr}_{1 *}\left(\mathcal{E} \smile \operatorname{pr}_{2}^{*} \alpha\right) \neq 0$. For each $i=0, \ldots, 2 k$, let $\gamma_{i, 1}, \ldots, \gamma_{i, j_{i}}$ be a basis of $H^{i}(X)$ and let $\eta_{i, 1}, \ldots, \gamma_{i, l_{i}}$ be a basis of $H^{i}(Z)$. 
Using the Künneth Isomorphism, we have

$$
\kappa^{-1}(\mathcal{E})=\sum_{i=0}^{2 k} \sum_{j=1}^{j_{i}} \sum_{l=1}^{l_{2 k-i}} a_{i, j, l} \gamma_{i, j} \otimes \eta_{2 k-i, l} .
$$

Since $\mathcal{E} \neq 0$, there is some $a_{i_{0}, j_{0}, l_{0}} \neq 0$. Since we are using field coefficients the cup product is a duality pairing; see [10, Ch. VI, Thm. 9.4]. We can therefore find some $\alpha \in H^{i_{0}}(Z)$ so that $\eta_{2 k-i_{0}, l_{0}} \smile \alpha=\left[z_{\bullet}\right]$ and $\eta_{2 k-i_{0}, l} \smile \alpha=0$ for every $l \neq l_{0}$. (Here, [z॰ $]$ is the fundamental cohomology class of a point $z_{\bullet} \in Z$ and a generator of $H^{2 k}(Z)$.) This implies that

$$
\begin{aligned}
\kappa^{-1}\left(\mathcal{E} \smile \operatorname{pr}_{2}^{*} \alpha\right) & =\sum_{i=0}^{2 k} \sum_{j=1}^{j_{i}} \sum_{l=1}^{l_{2 k-i}} a_{i, j, l} \gamma_{i, j} \otimes\left(\eta_{2 k-i, l} \smile \operatorname{pr}_{2}^{*} \alpha\right) \\
& =a_{i_{0}, j_{0}, l_{0}}\left(\gamma_{i_{0}, j_{0}} \otimes\left[z_{\bullet}\right]\right)+\sum_{i=i_{0}+1}^{2 k} \sum_{j=1}^{j_{i}} \sum_{l=1}^{l_{2 k-i}} a_{i, j, l} \gamma_{i, j} \otimes\left(\eta_{2 k-i, l} \smile \operatorname{pr}_{2}^{*} \alpha\right) .
\end{aligned}
$$

Lemma 2.3 implies

$$
\operatorname{pr}_{1 *}\left(\mathcal{E} \smile \operatorname{pr}_{2}^{*} \alpha\right)=a_{i_{0}, j_{0}, l_{0}} \gamma_{i_{0}, j_{0}} \neq 0 .
$$

we conclude that $f^{*} \circ g^{*}(\alpha) \neq(g \circ f)^{*}(\alpha)$.

\section{CRiteria FOR FunCtoriality}

We'll now start our study of the intersection $\rho_{1}^{-1}\left(\Gamma_{f}\right) \cap \rho_{3}^{-1}\left(\Gamma_{g}\right)$. Let (17) $U:=\left\{(x, y, z) \in X \times Y \times Z:(x, y) \in \Gamma_{f}, x \notin I_{f},(y, z) \in \Gamma_{g}\right.$, and $\left.y \notin I(g)\right\}$.

Lemma 5.1. We have

(i) $\rho_{1}^{-1}\left(\Gamma_{f}\right)$ and $\rho_{3}^{-1}\left(\Gamma_{g}\right)$ are smooth and intersect transversally at points of $U$ and

(ii) $V=\bar{U}$ is an irreducible component of $\rho_{1}^{-1}\left(\Gamma_{f}\right) \cap \rho_{3}^{-1}\left(\Gamma_{g}\right) \subset X \times Y \times Z$ that is mapped to $\Gamma_{\text {gof }}$ by $\rho_{2}$ with topological degree 1.

Consequently, if $U$ is dense in $\rho_{1}^{-1}\left(\Gamma_{f}\right) \cap \rho_{3}^{-1}\left(\Gamma_{g}\right)$ then $(g \circ f)^{*}=f^{*} \circ g^{*}$ on all cohomology.

We will call $V$ the principal component of $\rho_{1}^{-1}\left(\Gamma_{f}\right) \cap \rho_{3}^{-1}\left(\Gamma_{g}\right)$.

Proof. Since $x \notin I_{f}$ and $y \notin I_{g}, \rho_{1}^{*}\left(\Gamma_{f}\right)$ and $\rho_{3}^{*}\left(\Gamma_{g}\right)$ are smooth at any $(x, y, z) \in U$. For any $(x, y, z) \in U$ we have

$$
\begin{aligned}
& T_{(x, y, z)} \rho_{1}^{-1}\left(\Gamma_{f}\right)=\left\{\left(u_{1}, D f_{x} u_{1}, w_{1}\right): u_{1} \in T_{x} X \text { and } w_{1} \in T_{z} Z\right\} \text { and } \\
& T_{(x, y, z)} \rho_{3}^{-1}\left(\Gamma_{g}\right)=\left\{\left(u_{2}, v_{2}, D g_{y} v_{2}\right): v_{2} \in T_{y} Y \text { and } w_{2} \in T_{z} Z\right\} .
\end{aligned}
$$

Therefore, $T_{(x, y, z)} \rho_{1}^{-1}\left(\Gamma_{f}\right)+T_{(x, y, z)} \rho_{3}^{-1}\left(\Gamma_{g}\right)=T_{(x, y, z)} X \times Y \times Z$, so that $\rho_{1}^{-1}\left(\Gamma_{f}\right)$ and $\rho_{3}^{-1}\left(\Gamma_{g}\right)$ are transverse at $(x, y, z)$.

Notice that $\rho_{2}(U)$ is the graph of $(g \circ f)_{\mid X \backslash\left(I_{f} \cup f^{-1}(I(g))\right.}$, which is dense in $\Gamma_{g \circ f}$, by Lemma 3.3. Since $\rho_{2}$ is continuous and closed,

$$
\rho_{2}(V)=\rho_{2}(\bar{U})=\overline{\rho_{2}(U)}=\Gamma_{g \circ f} .
$$

Finally, notice that $\rho_{2}: U \rightarrow \rho_{2}(U)$ is one-to-one since for points of $U, x$ completely determines $y$ and $z$. In particular, since $\Gamma_{g \circ f}$ is irreducible, so is $V$. 
If $U$ is dense in $\rho_{1}^{-1}\left(\Gamma_{f}\right) \cap \rho_{3}^{-1}\left(\Gamma_{g}\right)$, then by Lemmas 2.5 and 2.7 we have

$$
\rho_{2 *}\left(\left[\rho_{1}^{-1}\left(\Gamma_{f}\right)\right] \smile\left[\rho_{3}^{-1}\left(\Gamma_{g}\right)\right]\right)=\rho_{2 *}([V])=\left[\Gamma_{g \circ f}\right] .
$$

It follows from Proposition 1.1 that $(g \circ f)^{*}=f^{*} \circ g^{*}$ on all cohomology.

Let us also prove one more helpful lemma:

Lemma 5.2. Let $X, Y, Z$ be algebraic manifolds of dimension $k$ and let $f: X \rightarrow Y$ and $g: Y \rightarrow Z$ be rational maps. If $\alpha \in H^{i}(Z)$ for $i \in\{0,1,2 k-1,2 k\}$, then $(g \circ f)^{*} \alpha=$ $\left(f^{*} \circ g^{*}\right) \alpha$.

Proof. Since $f$ is dominant, $f^{-1}\left(I_{g}\right) \cup I_{f}$ is a proper subvariety of $X$. Thus, any irreducible component of $\rho_{1}^{-1}\left(\Gamma_{f}\right) \cap \rho_{3}^{-1}\left(\Gamma_{g}\right)$ that projects under $\pi_{1} \circ \rho_{1}$ onto all of $X$ is equal to the principal component $V$. Similarly, since $g$ is dominant $g\left(I_{g} \cup f\left(I_{f}\right)\right)$ is a proper subvariety of $Z$, implying that any irreducible component of $\rho_{1}^{-1}\left(\Gamma_{f}\right) \cap \rho_{3}^{-1}\left(\Gamma_{g}\right)$ that projects under $\pi_{4} \circ \rho_{3}$ onto all of $Z$ is equal to the principal component $V$.

Thus, if $W \neq \Gamma_{g \circ f}$ is a $k$-dimensional subvariety of $\rho_{2}\left(\rho_{1}^{-1}\left(\Gamma_{f}\right) \cap \rho_{3}^{-1}\left(\Gamma_{g}\right)\right)$ whose fundamental class appears in the expression for $\rho_{2 *}\left(\rho_{1}^{*}\left[\Gamma_{f}\right] \cup \rho_{3}^{*}\left[\Gamma_{g}\right]\right)$, one finds that $\operatorname{dim}\left(\operatorname{pr}_{1}(W)\right) \leq$ $k-1$ and $\operatorname{dim}\left(\operatorname{pr}_{2}(W)\right) \leq k-1$. It then follows from Proposition 2.8 that if $i \in\{0,1,2 k-$ $1,2 k\}$ and $\alpha \in H^{i}(Z)$ then $\operatorname{pr}_{1 *}\left([W] \smile \operatorname{pr}_{2}^{*} \alpha\right)=0$. Equation (5) then implies that $(g \circ f)^{*} \alpha=\left(f^{*} \circ g^{*}\right) \alpha$.

We are now ready to prove Propositions 1.2 - 1.6. For the reader's convenience we'll repeat the statements before each of the proofs.

Proposition 1.2. Let $f: X \rightarrow Y$ and $g: Y \rightarrow Z$ be rational maps. Suppose that there exits an algebraic manifold $\widetilde{X}$ and holomorphic maps pr and $\widetilde{f}$ making the following diagram commute (wherever $f \circ$ pr is defined)

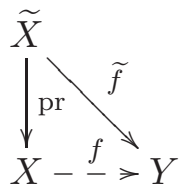

with the property that $\tilde{f}^{-1}(x)$ is a finite set for every $y \in Y$. Then, $(g \circ f)^{*}=f^{*} \circ g^{*}$ on all cohomology groups.

Proof. By Lemma 5.1, it suffices to show that $U$, given by (17), is dense in $\rho_{1}^{-1}\left(\Gamma_{f}\right) \cap \rho_{3}^{-1}\left(\Gamma_{g}\right)$.

Consider any $\left(x_{\bullet}, y_{\bullet}, z_{\bullet}\right) \in \rho_{1}^{-1}\left(\Gamma_{f}\right) \cap \rho_{3}^{-1}\left(\Gamma_{g}\right)$. We'll show that $\left(x_{\bullet}, y_{\bullet}, z_{\bullet}\right)$ is the limit of a sequence $\left\{\left(x_{n}, y_{n}, z_{n}\right)\right\} \subset U$. Since $f\left(I_{f}\right):=\pi_{2}\left(\pi_{1}^{-1}\left(I_{f}\right) \cap \Gamma_{f}\right)$ is a proper subvariety of $Y$, Lemma 3.3 gives that $\Gamma_{g}$ is the closure of the graph of $\left.g\right|_{Y \backslash\left(f\left(I_{f}\right) \cup I_{g}\right)}$. Therefore, we can choose a sequence

$$
\left\{\left(y_{n}, z_{n}\right)\right\} \in\left\{Y \times Z:(y, z) \in \Gamma_{g} \text { and } y \notin\left(f\left(I_{f}\right) \cup I_{g}\right)\right\}
$$

with $\left(y_{n}, z_{n}\right) \rightarrow\left(y_{\bullet}, z_{\bullet}\right)$.

Since $\left(x_{\bullet}, y_{\bullet}\right) \in \Gamma_{f}$, there exists $\widetilde{x}_{\bullet} \in \widetilde{X}$ with $\operatorname{pr}\left(\widetilde{x}_{\bullet}\right)=x_{\bullet}$ and $\widetilde{f}\left(\widetilde{x}_{\bullet}\right)=y_{\bullet}$. Since $\widetilde{f}$ is a finite map, it is open. Therefore we can choose a sequence of preimages $\widetilde{x}_{n}$ of $y_{n}$ under $\tilde{f}$ with $\widetilde{x}_{n} \rightarrow \widetilde{x}_{\bullet}$. If we let $x_{n}=\operatorname{pr}\left(\widetilde{x_{n}}\right)$, by continuity of pr we have $x_{n} \rightarrow x_{\bullet}$. Since $y_{n} \notin f\left(I_{f}\right)$ we have that each $x_{n} \notin I_{f}$. Therefore, we have found a sequence $\left(x_{n}, y_{n}, z_{n}\right) \in U$ with $\left(x_{n}, y_{n}, z_{n}\right) \rightarrow\left(x_{\bullet}, y_{\bullet}, z_{\bullet}\right)$. 
Proposition 1.4. (Diller-Favre [13, Prop. 1.13]) Let $X, Y$, and $Z$ be algebraic manifolds of dimension 2. Then $(g \circ f)^{*}=f^{*} \circ g^{*}$ if and only if there is no curve $C \subset X$ with $f\left(C \backslash I_{f}\right) \subset I_{g}$.

Remark 5.3. In the case that $(g \circ f)^{*} \neq f^{*} \circ g^{*}$, it follows from Lemma 5.2 that the discrepancy happens on $H^{2}(Z)$.

Proof. Suppose that there is no curve $C$ with $f\left(C \backslash I_{f}\right) \subset I_{g}$. By Lemma 5.1, it suffices to show that the set $U$, given by (17), is dense in $\rho_{1}^{-1}\left(\Gamma_{f}\right) \cap \rho_{3}^{-1}\left(\Gamma_{g}\right)$.

Let $\left(x_{\bullet}, y_{\bullet}, z_{\bullet}\right) \in \rho_{1}^{-1}\left(\Gamma_{f}\right) \cap \rho_{3}^{-1}\left(\Gamma_{g}\right)$. If $y_{\bullet} \notin I_{g}$, then we can choose a sequence $\left\{\left(x_{n}, y_{n}\right)\right\} \subset$ $\Gamma_{f}$ converging to $\left(x_{\bullet}, y_{\bullet}\right)$ with each $x_{n} \notin I_{f}$. Since $y_{\bullet} \notin I_{g}$ and $I_{g}$ is closed, $y_{n} \notin I_{g}$ for large enough $n$. Letting $z_{n}=g\left(y_{n}\right)$, we obtain a sequence $\left\{\left(x_{n}, y_{n}, z_{n}\right)\right\} \subset U$ which converges to $\left(x_{\bullet}, y_{\bullet}, z_{\bullet}\right)$.

Now, suppose $y_{\bullet} \in I_{g}$. As in the proof of Proposition 1.2 we will use that $\Gamma_{g}$ is the closure of the graph of $\left.g\right|_{Y \backslash\left(f\left(I_{f}\right) \cup I_{g}\right)}$. Therefore, we can choose a sequence $\left\{\left(y_{n}, z_{n}\right)\right\} \subset \Gamma_{g}$ with $\left(y_{n}, z_{n}\right) \rightarrow\left(y_{\bullet}, z_{\bullet}\right)$ and each $y_{n} \notin f\left(I_{f}\right) \cup I_{g}$. We must show that there is a sequence $x_{n} \in X \backslash I_{f}$ with $f\left(x_{n}\right)=y_{n}$ and $x_{n} \rightarrow x_{\bullet}$.

Since $X$ is a surface, we can make a resolution of indeterminacy of the form (6) where pr consists of a sequence of point blow-ups over $I_{f}$. Since $\left(x_{\bullet}, y_{\bullet}\right) \in \Gamma_{f}$, there exists $\left(\widetilde{x}_{\bullet}, y_{\bullet}\right) \in \Gamma_{\widetilde{f}}$ with $\operatorname{pr}\left(\widetilde{x}_{\bullet}\right)=x_{\bullet}$. Let $D$ be the component of $\widetilde{f}^{-1}\left(y_{\bullet}\right)$ containing $\widetilde{x}_{\bullet}$. Since $y_{\bullet} \neq f\left(C \backslash I_{g}\right)$ for any curve $C \subset X, \operatorname{pr}(D)=\left\{x_{\bullet}\right\}$.

Since $\widetilde{f}(D)=y_{\bullet}$ and $D$ is a component of $\widetilde{f}^{-1}\left(y_{\bullet}\right)$, we can choose a sequence $\widetilde{x_{n}} \in \widetilde{X}$ with $\widetilde{f}\left(\widetilde{x}_{n}\right)=\widetilde{y}_{n}$ such that $\widetilde{x_{n}} \rightarrow D$. Since $\operatorname{pr}(D)=x_{\bullet}$, the desired sequence $x_{n} \in X \backslash I_{f}$ is $x_{n}=\operatorname{pr}\left(\widetilde{x}_{n}\right)$.

Now, suppose that there are curves $C_{1}, \ldots, C_{m} \subset X$ with $\left\{y_{i}\right\}:=f\left(C_{i} \backslash I_{f}\right) \subset I_{g}$ for each $i$. For each $i, g\left(y_{i}\right)=D_{i}$ is a curve in $Z$. Then,

$$
\rho_{1}^{-1}\left(\Gamma_{f}\right) \cap \rho_{3}^{-1}\left(\Gamma_{g}\right)=V \cup \bigcup_{i=1}^{m} C_{i} \times\left\{y_{i}\right\} \times D_{i}
$$

with each term in the union being an independent irreducible component and $V=\bar{U}$ being the principal component.

Since each component has complex dimension 2, by Lemma 2.5

$$
\rho_{1}^{*}\left[\Gamma_{f}\right] \smile \rho_{3}^{*}\left[\Gamma_{g}\right]=[V]+\sum_{i=1}^{m} a_{i}\left[C_{i} \times\left\{y_{i}\right\} \times D_{i}\right],
$$

where each $a_{i}>0$ is a suitable intersection number.

By Lemmas 5.1 and 2.7,

$$
\rho_{2 *}\left([V]+\sum_{i=1}^{m} a_{i}\left[C_{i} \times\left\{y_{i}\right\} \times D_{i}\right]\right)=\left[\Gamma_{g \circ f}\right]+\sum_{i=1}^{m} a_{i}\left[C_{i} \times D_{i}\right] .
$$

Since $\sum_{i=1}^{m} a_{i}\left[C_{i} \times D_{i}\right] \neq 0$, it follows from Proposition 1.1 that $(g \circ f)^{*} \neq f^{*} \circ g^{*}$.

Remark 5.4. Up to this point, we have only needed the simple cases (i) and (ii) of Lemma 2.5 in which the subvarieties intersect with the correct dimension. The proofs of the criteria of Bedford-Kim and Dinh-Sibony below rely upon case (iii) of Lemma 2.5, 
since one can easily have components of $\rho_{1}^{-1}\left(\Gamma_{f}\right) \cap \rho_{3}^{-1}\left(\Gamma_{g}\right)$ of dimension $>k$. For example, if $f: \mathbb{C P}^{3} \rightarrow \mathbb{C P}^{3}$ and $g: \mathbb{C P}^{3} \rightarrow \mathbb{C P}^{3}$ are both Cremona involutions

$$
\left[x_{1}: x_{2}: x_{3}: x_{4}\right] \mapsto\left[x_{2} x_{3} x_{4}: x_{1} x_{3} x_{4}: x_{1} x_{2} x_{4}: x_{1} x_{2} x_{3}\right]
$$

then $\rho_{1}^{-1}\left(\Gamma_{1}\right) \cap \rho_{3}^{-1}\left(\Gamma_{g}\right)$ contains a four-dimensional component

$$
\left\{x_{0}=0\right\} \times\{[1: 0: 0: 0]\} \times\left\{z_{0}=0\right\} .
$$

Proposition 1.5. (Bedford-Kim [8, Thm. 1.1]) Let $X, Y$, and $Z$ be algebraic manifolds of dimension $k$. Let $f: X \rightarrow Y$ and $g: Y \rightarrow Z$ be rational maps. If there is no hypersurface $H$ with $f\left(H \backslash I_{f}\right) \subset I_{g}$, then $f^{*} \circ g^{*}=(g \circ f)^{*}$ on $H^{2}(Z)$.

Proof. By Lemmas 2.5 and 2.7,

$$
\rho_{2 *}\left(\rho_{1}^{*}\left[\Gamma_{f}\right] \smile \rho_{3}^{*}\left[\Gamma_{g}\right]\right)=\left[\Gamma_{g \circ f}\right]+\sum a_{i}\left[W_{i}\right]
$$

where each $W_{i}$ is a $k$-dimensional subvariety of $X \times Z$ and each $a_{i} \in \mathbb{Z}$. By Lemma 2.8, it suffices to show for every $i$ that $\operatorname{dim}\left(\operatorname{pr}_{1}\left(W_{i}\right)\right)<k-1$.

Suppose for some $i=i_{0}$ that $\operatorname{dim}\left(\operatorname{pr}_{1}\left(W_{i_{0}}\right)\right) \geq k-1$. Then, by commutativity of (4), $V_{i_{0}}:=\operatorname{pr}_{2}^{-1}\left(W_{i_{0}}\right)$ satisfies that $\operatorname{dim}\left(\pi_{1} \circ \rho_{1}\left(V_{i_{0}}\right)\right) \geq k-1$. The hypothesis that there is no hypersurface $H$ with $f\left(H \backslash I_{f}\right) \subset I_{g}$ implies that $\operatorname{dim}\left(f^{-1}\left(I_{g}\right)\right) \leq k-2$. Therefore $\operatorname{dim}\left(I_{f} \cup f^{-1}\left(I_{g}\right)\right) \leq k-2$. Thus, there is a dense set of points $(x, y, z) \in V_{i_{0}}$ with $x \notin$ $I_{f} \cup f^{-1}\left(I_{g}\right)$. All such points are in the principal component $V$; therefore $V=V_{i_{0}}$. This contradicts that $\rho_{2}\left(V_{i_{0}}\right)=W_{i_{0}} \neq \Gamma_{g \circ f}=\rho_{2}(V)$. We conclude that, $\operatorname{dim}\left(\operatorname{pr}_{1}\left(W_{i}\right)\right) \leq k-2$ for every $i$.

Remark 5.5. Recently, Bayraktar [4, Thm. 5.3] has proved that the Bedford-Kim criterion is necessary, i.e. if there is a hypersurface $H \subset X$ with $f\left(H \backslash I_{f}\right) \subset I_{g}$, then $(g \circ f)^{*} \neq f^{*} \circ g^{*}$ on $H^{2}(Z)$.

This does not seem to follow from the results developed in this note, since, when $k=$ $\operatorname{dim}(X) \geq 3, \rho_{1}^{-1}\left(\Gamma_{f}\right) \cap \rho^{-1}\left(\Gamma_{g}\right)$ may have components of dimension $>k$. (See Remark 5.4.) For this reason, the cup product $\rho_{1}^{*}\left[\Gamma_{f}\right] \smile \rho_{3}^{*}\left[\Gamma_{g}\right]$ may be represented by some $k$-dimensional subvarieties having negative coefficients. In particular, one must prove that the cohomology classes from all of the extra components of $\rho_{1}^{-1}\left(\Gamma_{f}\right) \cap \rho^{-1}\left(\Gamma_{g}\right)$ don't completely cancel.

Remark 5.6. There is an older criterion of Bedford and Kim [7, Prop. 1.2], which one can check is strictly weaker than the one stated in Proposition 1.5.

Recall that $\widetilde{\Sigma}_{f}^{\prime} \subset \Gamma_{f}$ is the set of points such that

(i) $\pi_{2}$ restricted to $\Gamma_{f}$ is not locally finite at $x$, and

(ii) $\pi_{2}((x, y)) \in I_{g}$ for every $(x, y) \in \widetilde{\Sigma}_{f}^{\prime}$.

Let $\Sigma_{f}^{\prime}:=\pi_{1}\left(\widetilde{\Sigma}_{f}^{\prime}\right)$.

Proposition 1.6. (Variant of Dinh-Sibony [16, Prop. 5.3.5]) Let $X, Y$, and $Z$ be algebraic manifolds of dimension $k$. Let $f: X \rightarrow Y$ and $g: Y \rightarrow Z$ be rational maps. If $\operatorname{dim} \Sigma_{f}^{\prime}<$ $k-p$, then $(g \circ f)^{*}=f^{*} \circ g^{*}$ on $H^{i}(Z)$ for $1 \leq i \leq 2 p$.

Proof. As in the proof of Proposition 1.5, $\rho_{2 *}\left(\rho_{1}^{*}\left[\Gamma_{f}\right] \smile \rho_{3}^{*}\left[\Gamma_{g}\right]\right)$ can be expressed by (21). By Lemma 2.8 it suffices to show for every $i$ that $\operatorname{dim}\left(\operatorname{pr}_{1}\left(W_{i}\right)\right)<k-p$.

Suppose for some $i=i_{0}$ that $\operatorname{dim}\left(\operatorname{pr}_{1}\left(W_{i_{0}}\right)\right) \geq k-p$. Then, by commutativity of (4), $V_{i_{0}}:=\operatorname{pr}_{2}^{-1}\left(W_{i_{0}}\right)$ satisfies that $\operatorname{dim}\left(\pi_{1} \circ \rho_{1}\left(V_{i_{0}}\right)\right) \geq k-p$. We'll show that the set $U$, given 
by (17), is dense in $V_{i_{0}}$, implying that $V_{i_{0}}=V$. This will contradict that $\rho_{2}\left(V_{i_{0}}\right)=W_{i_{0}} \neq$ $\Gamma_{g \circ f}=\rho_{2}(V)$.

Since $\operatorname{dim}\left(\Sigma_{f}^{\prime}\right)<k-p$, we have that $V_{i_{0}} \backslash\left(\pi_{1} \circ \rho_{1}\right)^{-1}\left(\Sigma_{f}^{\prime}\right)$ is dense in $V_{i_{0}}$. Therefore, it suffices to show that $U$ is dense in $V_{i_{0}} \backslash\left(\pi_{1} \circ \rho_{1}\right)^{-1}\left(\Sigma_{f}^{\prime}\right)$. Let

$$
\left(x_{\bullet}, y_{\bullet}, z_{\bullet}\right) \in V_{i_{0}} \backslash\left(\pi_{1} \circ \rho_{1}\right)^{-1}\left(\Sigma_{f}^{\prime}\right) .
$$

First suppose that $y_{\bullet} \notin I_{g}$. Then, we can choose a sequence $\left(x_{n}, y_{n}\right) \in \Gamma_{f}$ with $x_{n} \notin I_{f}$ converging to $\left(x_{\bullet}, y_{\bullet}\right)$. Since $y_{\bullet} \notin I_{g}$ and $I_{g}$ is closed, $y_{n} \notin I_{g}$ for large enough $n$. Thus, if we let $z_{n}=g\left(y_{n}\right)$, we obtain a sequence $\left(x_{n}, y_{n}, z_{n}\right) \in U$ that converges to $\left(x_{\bullet}, y_{\bullet}, z_{\bullet}\right)$.

Now suppose that $y_{\bullet} \in I_{f}$. By Lemma 3.3, $\Gamma_{g}$ is the closure of the graph of $\left.g\right|_{Y \backslash\left(I_{g} \cup f\left(I_{f}\right)\right)}$. Thus, we can find a sequence $\left(y_{n}, z_{n}\right)$ in the graph of $\left.g\right|_{Y \backslash\left(I_{g} \cup f\left(I_{f}\right)\right)}$ with $\left(y_{n}, z_{n}\right) \rightarrow\left(y_{\bullet}, z_{\bullet}\right)$. Meanwhile, since $x_{\bullet} \notin \Sigma_{f}^{\prime}$ and $y_{\bullet} \in I_{g}, \pi_{2} \mid \Gamma_{f}$ is a finite map in a neighborhood of $\left(x_{\bullet}, y_{\bullet}\right)$. It follows from the Weierstrass Preparation Theorem that $\left.\pi_{2}\right|_{\Gamma_{f}}$ is an open map in that neighborhood. Therefore, there is a sequence $x_{n} \in X$ with $\left(x_{n}, y_{n}\right) \in \Gamma_{f}$ and $\left(x_{n}, y_{n}\right) \rightarrow\left(x_{\bullet}, y_{\bullet}\right)$. Since $y_{n} \notin f\left(I_{f}\right), x_{n} \notin I_{f}$. Thus, we have found a sequence $\left\{\left(x_{n}, y_{n}, z_{n}\right)\right\} \subset U$ with $\left(x_{n}, y_{n}, z_{n}\right) \rightarrow\left(x_{\bullet}, y_{\bullet}, z_{\bullet}\right)$. We conclude that $U$ is dense in $V_{i_{0}}$.

Remark 5.7. The reader who is interested in proving Proposition 1.6 using currents should note that Truong [35] presents an approach to pulling back $(p, p)$-currents for $p>1$ that is somewhat different from [16]. In particular, one can also use Theorem 7 from [35] to prove Proposition 1.6.

\section{A cautionary example}

In this section, we present a rational map $f: X \rightarrow X$ of a three-dimensional algebraic manifold $X$ that is not 2-stable, but has the property that $\left(\left.f\right|_{X \backslash I_{f}}\right)^{-1}(x)$ is a finite set for every $x \in X$.

Let $f_{0}: \mathbb{P}^{3} \rightarrow \mathbb{P}^{3}$ be the composition $f_{0}=\alpha_{0} \circ s_{0}$, where $\alpha_{0}$ is the birational map

$$
\begin{aligned}
& \alpha_{0}\left(\left[x_{1}: x_{2}: x_{3}: x_{4}\right]\right) \\
& =\left[x_{1}\left(x_{2}-x_{4}\right)\left(x_{3}-x_{4}\right): x_{4}\left(x_{2}-x_{4}\right)\left(x_{3}-x_{4}\right): x_{4}\left(x_{2}-x_{1}\right)\left(x_{3}-x_{4}\right): x_{4}\left(x_{2}-x_{4}\right)\left(x_{3}-x_{1}\right)\right]
\end{aligned}
$$

and $s_{0}$ is the squaring map

$$
s_{0}\left(\left[x_{1}: x_{2}: x_{3}: x_{4}\right]\right)=\left[x_{1}^{2}: x_{2}^{2}: x_{3}^{2}: x_{4}^{2}\right] .
$$

Let $\varrho: X \rightarrow \mathbb{P}^{3}$ be the blow-up of $\mathbb{P}^{3}$ at the five points $[1: 0: 0: 0],[0: 1: 0: 0],[0: 0: 1:$ $0],[0: 0: 0: 1]$, and $[1: 1: 1: 1]$ and let $\alpha: X \rightarrow X, s: X \rightarrow X$, and $f$ be the lifts of $\alpha_{0}$, $s_{0}$, and $f_{0}$.

Proposition 6.1. $f: X \rightarrow X$ satisfies for every $x \in X$ that $\left(\left.f\right|_{X \backslash I_{f}}\right)^{-1}(x)$ is a finite set, but $f$ is not 2-stable.

Proof. We will start by showing that $\left(\left.f\right|_{X \backslash I_{f}}\right)^{-1}(x)$ is a finite set for every $x \in X$. One can check that $\alpha_{0}: \mathbb{P}^{3} \rightarrow \mathbb{P}^{3}$ satisfies

$$
\begin{aligned}
I_{\alpha_{0}}=\quad & \left\{x_{1}=x_{4}=0\right\} \cup\left\{x_{2}=x_{4}=0\right\} \cup\left\{x_{3}=x_{4}=0\right\} \\
& \cup\left\{x_{1}=x_{2}=x_{4}\right\} \cup\left\{x_{1}=x_{3}=x_{4}\right\} \cup\left\{x_{2}=x_{3}=x_{4}\right\} .
\end{aligned}
$$


The four points $[1: 0: 0: 0],[0: 1: 0: 0],[0: 0: 1: 0],[1: 1: 1: 1]$ where these six lines meet are each blown-up up by $\alpha_{0}$ to the following hyperplanes

$$
\left\{x_{2}=0\right\},\left\{x_{2}=x_{3}\right\},\left\{x_{2}=x_{4}\right\} \text {, and }\left\{x_{1}=x_{2}\right\},
$$

respectively. Once these four points are removed, the six lines in $I_{\alpha_{0}}$ map by flip indeterminacy (see, for example, [30]) to the lines

$$
\begin{aligned}
& \left\{x_{2}=x_{3}=x_{4}\right\} \cup\left\{x_{2}=x_{4}=0\right\} \cup\left\{x_{2}=x_{3}=0\right\} \\
& \cup\left\{x_{1}=x_{2}=x_{4}\right\} \cup\left\{x_{1}=x_{2}=x_{3}\right\} \cup\left\{x_{1}=x_{2}=x_{4}\right\},
\end{aligned}
$$

respectively. A flip indeterminacy from a line $L_{1}$ to a line $L_{2}$ blows up any point $p \in L_{1}$ to all of $L_{2}$, based on the direction that one approaches $p$ within a transversal plane. Meanwhile, there is a collapsing behavior: if one approaches any two points of $L_{1}$ with the same transversal direction, one is sent by $f$ to the same point of $L_{2}$.

The critical set of $\alpha_{0}$ consists of the hypersurfaces

$$
\left\{x_{4}=0\right\} \cup\left\{x_{1}=x_{4}\right\} \cup\left\{x_{2}=x_{4}\right\} \cup\left\{x_{3}=x_{4}\right\},
$$

which are all collapsed by $\alpha_{0}$ to the points $[1: 0: 0: 0],[1: 1: 1: 1],[0: 0: 1: 0]$, and $[0: 0: 0: 1]$, respectively, with the first three of these points in $I_{\alpha_{0}}$.

When creating $X$, we have blown-up each of the images of the varieties that are collapsed by $\alpha_{0}$ as well as $[0: 0: 0: 1]=\alpha_{0}^{-1}([0: 1: 0: 0])$. Using the universal property of blow-ups [17] (or direct calculations), one can check the indeterminacy set of $\alpha: X \rightarrow X$ is the proper transform of $I_{\alpha_{0}}$ and that $\alpha$ has no critical points (outside of $I_{\alpha}$ ). In particular, $\alpha$ collapses no curves or hypersurfaces lying outside of $I_{\alpha}$.

One can check that $s: X \rightarrow X$ is holomorphic in a neighborhood of each of the exceptional divisors, inducing the squaring map on each of the exceptional divisors $E_{\text {[1:0:0:0] }}$, $E_{[0: 1: 0: 0]}, E_{[0: 0: 1: 0]}, E_{[0: 0: 0: 1]}$ and is the identity on the exceptional divisor $E_{\text {[1:1:1:1] }}$. As a result, the only indeterminacy points of $s$ are the lifts under $\varrho$ above the 7 points in $s_{0}^{-1}([1: 1: 1: 1]) \backslash[1: 1: 1: 1]$, each of which is blown-up by $s$ to $E_{[1: 1: 1: 1]}$. Since $s_{0}$ did not collapse and hypersurfaces or curves and $s$ does not collapse anything in these exceptional divisors, we conclude that $s$ also doesn't collapse and hypersurfaces or curves.

The indeterminacy of $f=\alpha \circ s$ is contained in $s^{-1}\left(I_{\alpha}\right) \cup I_{s}$. One can check that $I_{s} \subset I_{f}$ since $\alpha$ doesn't collapse $E_{\text {[1:1:1:1] }}$. Meanwhile, $s^{-1}\left(I_{\alpha}\right)$ is the proper transform of the 15 lines

$$
\begin{aligned}
s_{0}^{-1}\left(I_{\alpha_{0}}\right)=\quad & \left\{x_{1}=x_{4}=0\right\} \cup\left\{x_{2}=x_{4}=0\right\} \cup\left\{x_{3}=x_{4}=0\right\} \\
& \cup\left\{x_{1}= \pm x_{2}= \pm x_{4}\right\} \cup\left\{x_{1}= \pm x_{3}= \pm x_{4}\right\} \cup\left\{x_{2}= \pm x_{3}= \pm x_{4}\right\} .
\end{aligned}
$$

Taking any point from one of these 15 lines that is not on one of the exceptional divisors, one can use the homogeneous expression for $f_{0}$ to see that each such point is in $I_{f}$. Therefore, $I_{f}=s^{-1}\left(I_{\alpha}\right) \cup I_{s}$. Since neither $\alpha$ nor nor $s$ collapse any variety outside of their indeterminate sets, we conclude that $f$ doesn't collapse any variety outside of $I_{f}$.

The only non-trivial cohomology groups of $X$ are

$$
H^{0}(X) \cong \mathbb{C}, H^{2}(X)=H^{(1,1)}(X) \cong \mathbb{C}^{6}, H^{4}(X)=H^{(2,2)}(X) \cong \mathbb{C}^{6}, \text { and } H^{6}(X) \cong \mathbb{C} .
$$

By Lemma 5.2, any rational map acts stably on $H^{0}(X)$ and $H^{6}(X)$. Since $I_{f}$ is of codimension $\geq 2$ and $f$ collapses nothing outside of $I_{f}$, the Bedford-Kim criterion (Prop. 1.5) implies that $f$ acts stably on $H^{(1,1)}(X)$. Therefore, in order to prove that $f$ is not 2-stable, it suffices to show that $\left(f^{2}\right)^{*} \neq\left(f^{*}\right)^{2}$ on $H^{*}(X)$. 
For notational convenience, we'll write the composition as $g \circ f$, where $X=Y=Z$ and $f: X \rightarrow Y$ and $g: Y \rightarrow Z$ are the same map. We will first show that every component of $\rho_{1}^{-1}\left(\Gamma_{f}\right) \cap \rho_{3}^{-1}\left(\Gamma_{g}\right)$ has the correct dimension $(=3)$, so that Lemma 2.5 implies that each component appears with positive multiplicity in the cup product $\rho_{1}^{*}\left(\left[\Gamma_{f}\right]\right) \smile \rho_{3}^{*}\left(\left[\Gamma_{g}\right]\right)$. We will then show that there is at least one component $V^{\prime}$ of the intersection other than the principal component $V$ (see Lemma 5.1 for the definition of the principal component) with the property that $\rho_{2 *}\left(\left[V^{\prime}\right]\right) \neq 0$. Non-functoriality $\left(f^{2}\right)^{*} \neq\left(f^{*}\right)^{2}$ will then follow from Proposition 1.1.

Consider a component $V^{\prime} \neq V$ of $\rho_{1}^{-1}\left(\Gamma_{f}\right) \cap \rho_{3}^{-1}\left(\Gamma_{g}\right)$. Since $V^{\prime} \neq V$ and $f$ a finite map outside of $I_{f},\left(\pi_{1} \circ \rho_{1}\right)\left(V^{\prime}\right) \subset I_{f}$. Meanwhile, $\left(\pi_{2} \circ \rho_{1}\right)\left(V^{\prime}\right) \subset I_{g}$. Let $\phi: V^{\prime} \rightarrow \phi\left(V^{\prime}\right) \subset X \times Y$ be the restriction of the projection onto the first two coordinates. If $\operatorname{dim}\left(V^{\prime}\right) \geq 4$, then, since $\operatorname{dim}\left(\phi\left(V^{\prime}\right)\right) \leq 2$, the fibers over generic points satisfy $\operatorname{dim}\left(\phi^{-1}(x, y)\right) \geq 2$. However, there are finitely many points $y \in Y$ with $\operatorname{dim}(g(y)) \geq 2$, implying that $\operatorname{dim}\left(\pi_{2} \circ \rho_{1}\right)\left(V^{\prime}\right)=0$. Therefore, the dimension of the projections of $V^{\prime}$ onto $X, Y$, and $Z$ would be 1,0 , and 2 , respectively, implying that $\operatorname{dim}\left(V^{\prime}\right)=3$.

We will now find a component $V^{\prime} \neq V \subset \rho_{1}^{-1}\left(\Gamma_{f}\right) \cap \rho_{3}^{-1}\left(\Gamma_{g}\right)$ so that $\rho_{2 *}\left(\left[V^{\prime}\right]\right) \neq 0$. Let $L_{1} \subset X$ be the proper transform of $\left\{x_{1}=x_{3}=x_{4}\right\}$, let $L_{2} \subset Y$ be the proper transform of $\left\{y_{1}=y_{2}=y_{3}\right\}$, let $p=\varrho^{-1}([1: 1: 1:-1]) \in L_{2}$, and let $H \subset Z$ be the proper transform of $\left\{z_{1}=z_{2}\right\}$. Since $s$ maps a neighborhood of $L_{1}$ biholomorphically onto a neighborhood $L_{1}$ and $\alpha$ blows-up each point of $L_{1}$ to all of $L_{2}$, we have that $L_{1} \times\{p\} \subset \Gamma_{f}$. Meanwhile, since $s$ blows up the indeterminate point $p$ to $E_{[1: 1: 1: 1]}$, which is mapped by $\alpha$ to the plane $z_{1}=z_{2}$, we have that $\{p\} \times H \subset \Gamma_{g}$. We conclude that

$$
V^{\prime}:=L_{1} \times\{p\} \times H \subset \rho_{1}^{-1}\left(\Gamma_{f}\right) \cap \rho_{3}^{-1}\left(\Gamma_{g}\right) .
$$

Since $V^{\prime}$ is an irreducible 3 -dimensional variety with $\left(\pi_{1} \circ \rho_{1}\right)(V)=L_{1} \subsetneq X$, it is not the principal component $V$. It is the collapsing behavior of the flip indeterminacy along $L_{1}$ into the point of indeterminacy $p$ that produces this extra component of $\rho_{1}^{-1}\left(\Gamma_{f}\right) \cap \rho_{3}^{-1}\left(\Gamma_{g}\right)$. This will lead to non-functoriality of the composition.

Since $\rho_{2}$ maps $V^{\prime}$ biholomorphically to $L_{1} \times H \subset X \times Z, \rho_{2 *}\left(\left[V^{\prime}\right]\right) \neq 0$. We conclude that $(g \circ f)^{*} \neq f^{*} \circ g^{*}$.

Remark 6.2. In a joint work with S. Koch [29], we check that $f$ can be lifted to a further blow-up of $X$ on which Proposition 1.2 can be applied. We then compute that the first and second dynamical degrees of this lift satisfy that $\lambda_{1} \approx 2.3462$ is the largest root of

$$
p_{1}(z)=z^{4}-z^{3}-4 z-8,
$$

$\lambda_{2} \approx 4.6658$ is the largest root of

$$
p_{2}(z)=z^{9}-3 z^{8}-16 z^{6}-192 z^{5}+384 z^{4}+128 z^{3}+6144 z-8192 .
$$

Since dynamical degrees are invariant under birational conjugacy, these are the same as the dynamical degrees of $f: X \rightarrow X$. However, one can check that $\operatorname{dim}\left(H^{4}(X)\right)=6$. Since $p_{2}$ is an irreducible polynomial of degree $9>6$, this gives an alternate proof that $f: X \rightarrow X$ is not 2 -stable.

Question 6.3. Does there exist a rational map $f: \mathbb{P}^{3} \rightarrow \mathbb{P}^{3}$ such that $\left(\left.f\right|_{\mathbb{P}^{3} \backslash I_{f}}\right)^{-1}(x)$ is a finite set for every $x \in \mathbb{P}^{3}$, that is not 2-stable? 


\section{ACKNowledgements}

Much of this work is inspired by the techniques from [2] and by the results of the other authors mentioned above. We have also benefited from discussions with Eric Bedford, Olguta Buse, Jeffrey Diller, Kyounghee Kim, Sarah Koch, Patrick Morton, and Tuyen Truong. Finally, we thank the referees for their several helpful suggestions. This work is supported in part by the NSF grant DMS-1102597 and startup funds from the Department of Mathematics at IUPUI.

\section{REFERENCES}

[1] Macaulay2 computer software. http://www.math.uiuc.edu/Macaulay2/.

[2] E. Amerik, A computation of invariants of a rational self-map, Ann. Fac. Sci. Toulouse Math. (6) 18 (2009), no. 3, 445-457.

[3] - Existence of non-preperiodic algebraic points for a rational self-map of infinite order, Math. Res. Lett. 18 (2011), no. 2, 251-256.

[4] T. Bayraktar, Green currents for meromorphic maps of compact Kähler manifolds, Journal of Geometric Analysis (2012) 1-29.

[5] E. Bedford, On the dynamics of birational mappings of the plane, J. Korean Math. Soc. 40 (2003), no. 3, 373-390.

[6] — The Dynamical Degrees of a Mapping, in Proceedings of the International Workshop Future Directions in Difference Equations, 3-14, Publicacións da Universidade de Vigo, Vigo, Spain (2011).

[7] E. Bedford and K. Kim, On the degree growth of birational mappings in higher dimension, J. Geom. Anal. 14 (2004), no. 4, 567-596.

[8] - Degree Growth of Matrix Inversion: Birational Maps of Symmetric, Cyclic Matrices, Discrete Contin. Dyn. Syst. 21 (2008), no. 4, 977-1013.

[9] A. Bonifant and J. E. Fornaess, Growth of Degree for Iterates of Rational Maps in Several Variables, Indiana Univ. Math. J. 49 (2000), no. 2, 751-778.

[10] G. E. Bredon, Topology and geometry, Vol. 139 of Graduate Texts in Mathematics, Springer-Verlag, New York (1993), ISBN 0-387-97926-3.

[11] E. M. Chirka, Complex analytic sets, Vol. 46 of Mathematics and its Applications (Soviet Series), Kluwer Academic Publishers Group, Dordrecht (1989), ISBN 0-7923-0234-6. Translated from the Russian by R. A. M. Hoksbergen.

[12] J. Diller, R. Dujardin, and V. Guedj, Dynamics of meromorphic maps with small topological degree I: from cohomology to currents, Indiana Univ. Math. J. 59 (2010), no. 2, 521-561.

[13] J. Diller and C. Favre, Dynamics of bimeromorphic maps of surfaces, Amer. J. Math. 123 (2001), no. 6, 1135-1169.

[14] T.-C. Dinh, V.-A. Nguyen, and T. T. Truong, Equidistribution for meromorphic maps with dominant topological degree. Preprint: http://arxiv.org/abs/1303.5992.

[15] T.-C. Dinh and N. Sibony, Une borne supérieure pour l'entropie topologique d'une application rationnelle, Ann. of Math. (2) 161 (2005), no. 3, 1637-1644.

[16] - Super-potentials of positive closed currents, intersection theory and dynamics, Acta Math. 203 (2009), no. 1, 1-82.

[17] D. Eisenbud and J. Harris, The geometry of schemes, Vol. 197 of Graduate Texts in Mathematics, Springer-Verlag, New York (2000), ISBN 0-387-98638-3; 0-387-98637-5.

[18] C. Favre and E. Wulcan, Degree growth of monomial maps and McMullen's polytope algebra, Indiana Univ. Math. J. 61 (2012) 493-524.

[19] J. E. Fornaess and N. Sibony, Complex dynamics in higher dimension. II, in Modern methods in complex analysis (Princeton, NJ, 1992), Vol. 137 of Ann. of Math. Stud., 135-182, Princeton Univ. Press, Princeton, NJ (1995).

[20] S. Friedland, Entropy of polynomial and rational maps, Ann. of Math. (2) 133 (1991), no. 2, 359-368.

[21] W. Fulton, Introduction to intersection theory in algebraic geometry, Vol. 54 of CBMS Regional Conference Series in Mathematics, Published for the Conference Board of the Mathematical Sciences, Washington, DC (1984), ISBN 0-8218-0704-8. 
[22] - Intersection theory, Vol. 2 of Ergebnisse der Mathematik und ihrer Grenzgebiete. 3. Folge. A Series of Modern Surveys in Mathematics [Results in Mathematics and Related Areas. 3rd Series. A Series of Modern Surveys in Mathematics], Springer-Verlag, Berlin, second edition (1998), ISBN 3-54062046-X; 0-387-98549-2.

[23] P. Griffiths and J. Harris, Principles of algebraic geometry, Wiley Classics Library, John Wiley \& Sons Inc., New York (1994), ISBN 0-471-05059-8. Reprint of the 1978 original.

[24] V. Guedj, Entropie topologique des applications méromorphes, Ergodic Theory Dynam. Systems 25 (2005), no. 6, 1847-1855.

[25] — Ergodic properties of rational mappings with large topological degree, Ann. of Math. (2) 161 (2005), no. 3, 1589-1607.

[26] R. Hartshorne, Algebraic geometry, Springer-Verlag, New York (1977), ISBN 0-387-90244-9. Graduate Texts in Mathematics, No. 52.

[27] M. Jonsson and E. Wulcan, Canonical heights for plane polynomial maps of small topological degree, Math. Res. Lett. 19 (2012), no. 6, 1207-1217.

[28] S. Kawaguchi and J. H. Silverman, On the dynamical and arithmetic degrees of rational self-maps of algebraic varieties. Preprint: http://arxiv.org/abs/1208.0815.

[29] S. Koch and R. K. Roeder, Computing Dynamical Degrees for a family of maps of the Deligne-Mumford compactification of $\mathcal{M}_{0, n}$. In preparation.

[30] J. Kollár and S. Mori, Birational geometry of algebraic varieties, Vol. 134, Cambridge University Press (2008).

[31] J.-L. Lin, Pulling back cohomology classes and dynamical degrees of monomial maps, Bull. Soc. Math. France 140 (2012), no. 4, 533-549 (2013).

[32] J.-L. Lin and E. Wulcan, Stabilization of monomial maps in higher codimension. To appear in Annales de l'Institut Fourier; available at http://arxiv.org/abs/1206.4925.

[33] A. Russakovskii and B. Shiffman, Value distribution for sequences of rational mappings and complex dynamics, Indiana Univ. Math. J. 46 (1997), no. 3, 897-932.

[34] J. H. Silverman, Dynamical degree, arithmetic entropy, and canonical heights for dominant rational self-maps of projective space. http://dx.doi.org/10.1017/etds.2012.144.

[35] T. T. Truong, Pull-back of currents by meromorphic maps. To appear in Bulletin de la Société Mathématique de France; available at http://arxiv.org/abs/1107.1743.

[36] - The simplicity of the first spectral radius of a meromorphic map. Preprint: http://arxiv.org/ abs/1212.1091.

[37] C. Voisin, Hodge theory and complex algebraic geometry. I, Vol. 76 of Cambridge Studies in Advanced Mathematics, Cambridge University Press, Cambridge, english edition (2007), ISBN 978-0-521-71801-1. Translated from the French by Leila Schneps.

[38] — Hodge theory and complex algebraic geometry. II, Vol. 77 of Cambridge Studies in Advanced Mathematics, Cambridge University Press, Cambridge, english edition (2007), ISBN 978-0-521-71802-8. Translated from the French by Leila Schneps.

Department of Mathematical Sciences, IUPUi, LD Building, Room 224Q, 402 North BlackFord Street, Indianapolis, Indiana 46202-3267, United States

E-mail address: rroeder@math.iupui.edu 